\title{
An infant with pneumonia, failure to thrive and persistent radiographical changes
}

\author{
N. Thompson, S. Saglani and A. Bush
}

\section{CASE HISTORY}

A 9-month-old male presented to his local hospital with a 2week history of diarrhoea and vomiting and a 48-h history of tachypnoea and grunting. He was afebrile on admission and oxygen saturation was $96 \%$ in room air, but he had mild respiratory distress and tachypnoea. Inflammatory markers were not raised but a chest radiograph showed left-sided collapse and consolidation. Treatment was commenced with i.v. antibiotics. The patient's respiratory pattern worsened $24 \mathrm{~h}$ after admission and his oxygen saturation dropped to $90 \%$, requiring $2.5 \mathrm{~L} \cdot \mathrm{min}^{-1}$ supplemental oxygen. His diarrhoea settled after $48 \mathrm{~h}$ but he continued to have intermittent vomiting.

The patient was an only child, born at term to unrelated parents and had no significant illnesses until this episode. He had a complete immunisation history and was achieving his developmental milestones normally. Weight gain was along the 50th centile for the first 6 months but had dropped down towards the second centile since weaning had occurred. There was no other family history of note.

After 2.5 weeks, he still had a significant supplemental oxygen requirement and persisting left-sided chest radiograph abnormalities, despite a change to broader spectrum antibiotics, and so was transferred to the Royal Brompton Hospital (London, UK) for a respiratory opinion. On arrival, he had no significant fever and over the next 4 days he became normally saturated in room air. Antibiotics were stopped as his clinical condition improved.

Initial investigations on presentation were normal (table 1), including white cell count differential and blood film. Early morning gastric washings were negative, as were Mantoux and sweat tests. Blood, urine and stool cultures, nasopharyngeal aspirate (NPA) for viral immunofluorescence and mycoplasma

\section{TABLE 1 Initial investigations at presentation}

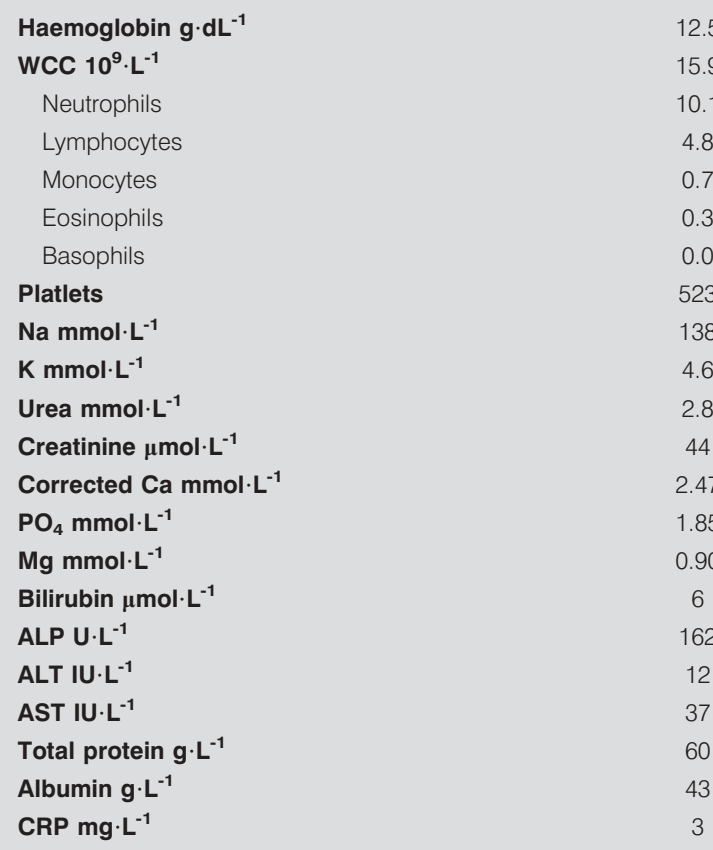

WCC: white cell count; ALP: alkaline phosphatase; ALT: alanine transaminase; AST: aspartate aminotransferase; CRP: C-reactive protein.

serology were all negative. Radioallergosorbent test for common allergens was negative as was a coeliac screen.

The patient remained mildly tachypnoeic, despite the absence of an oxygen requirement. A chest radiograph was taken at this time (fig. 1). Following this a high-resolution computed tomography (HRCT) scan was performed (fig. 2). 


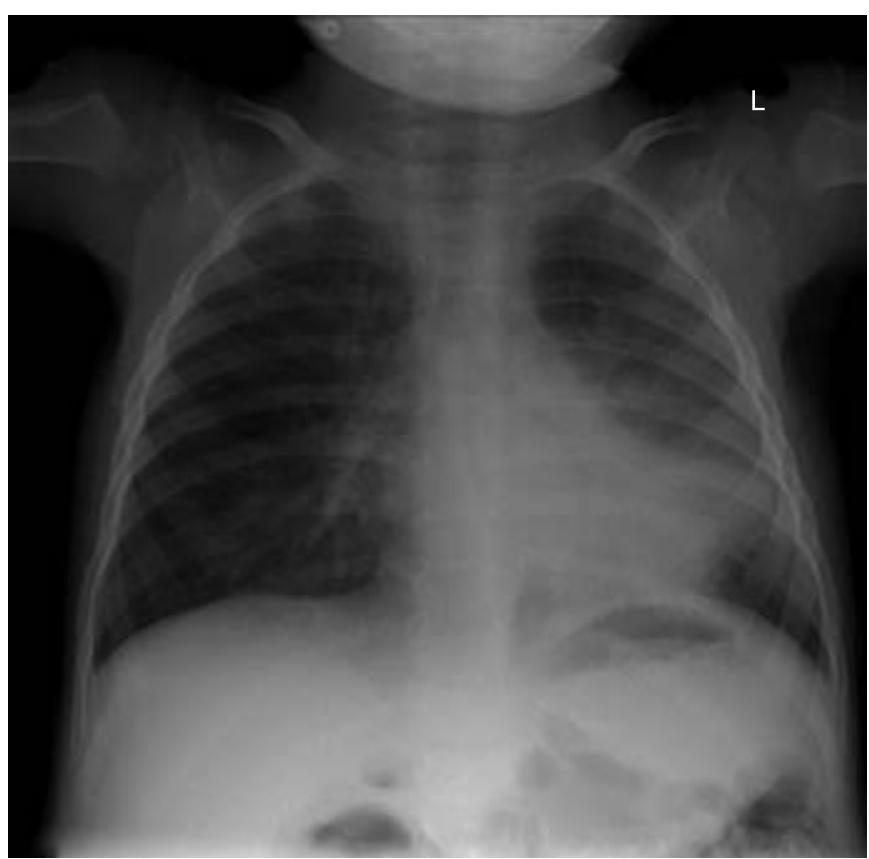

FIGURE 1. Chest radiograph 3 weeks into the illness.

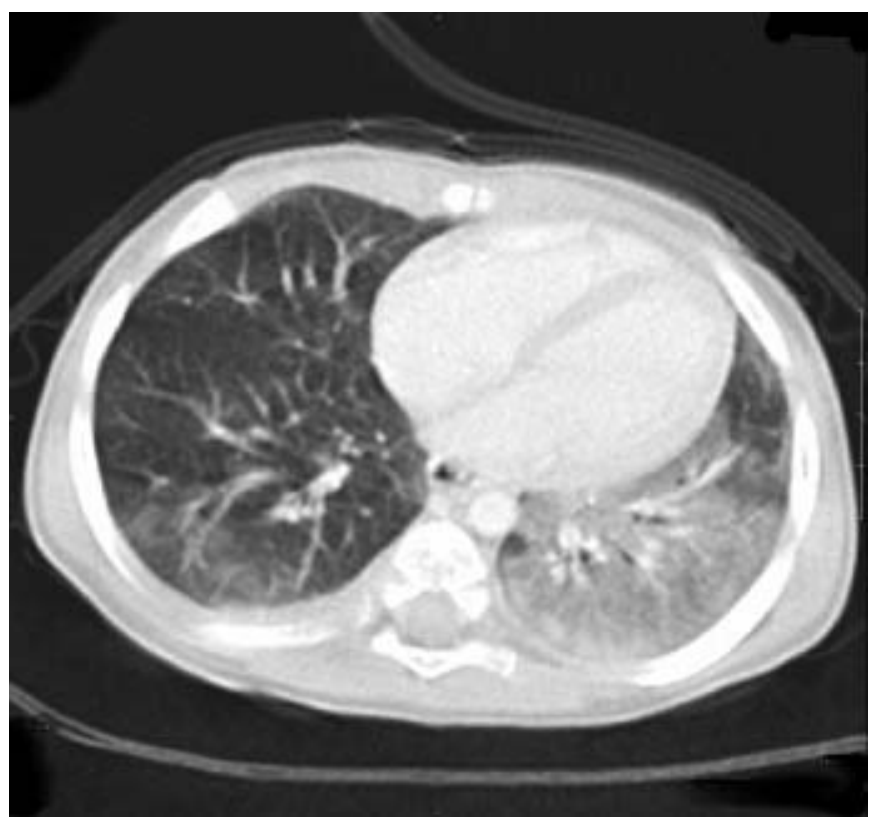

FIGURE 2. High-resolution computed tomography of the thorax, 3 weeks into the illness.

BEFORE TURNING THE PAGE, INTERPRET THE PATIENT'S HISTORY, CHEST RADIOGRAPH AND HRCT SCAN, AND SUGGEST A DIAGNOSIS. 


\section{INTERPRETATION}

\section{Chest radiograph}

There is increased density behind the left heart together with consolidation in the left midzone, in particular the lingula. These findings are consistent with extensive left-sided consolidation.

\section{HRCT}

The patent airways can be followed out to at least segmental level. There was no hilar lymphadenopathy. There is extensive parenchymal abnormality throughout much of the left lung. Although there are small areas of consolidation, much of the infiltrate is of ground-glass attenuation with air bronchograms and vascular markings clearly identifiable within the infiltrate. This is quite unlike true consolidation, in which vascular markings would not be seen. The mediastinal structures appear normal.

\section{Bronchoscopy}

Due to the prolonged clinical course and atypical HRCT changes, fibreoptic bronchoscopy was performed, despite the fact that the respiratory illness appeared to be improving. This was essentially normal, with minimal inflammation only. There were moderate amounts of secretions from the left lower lobe. Bronchoalveolar lavage (BAL) cytology showed neutrophilia, with a few fat-laden macrophages and a few foamy macrophages (table 2). There was no growth on culture.

Immunofluorescence was positive for Pneumocystis jiroveci pneumonia (PCP) but negative for other respiratory pathogens. A $\mathrm{pH}$ probe was placed in the mid-oesophagus at the time of bronchoscopy. The trace showed severe gastro-oesophogeal reflux, with $\mathrm{pH}<4$ for $22 \%$ of the duration of the study. Aspiration of gastric acid seems unlikely, given the small numbers of fat-laden macrophages and the absence of typical changes on the HRCT scan, but cannot be excluded.

\section{Diagnosis: PCP pneumonia and gastro-oesophogeal reflux}

\section{CLINICAL COURSE}

Following the discovery of PCP in the BAL, the patient was further investigated for immunodeficiency (table 3). HIV testing was negative but total immunoglobulin (Ig) and IgG subclass levels were all very low, as were his antibody responses to diphtheria and tetanus vaccines. Although lymphocyte numbers were normal, evaluation of subsets revealed low levels of T-cells, and a provisional diagnosis of severe combined immunodeficiency (SCID) was made. He was treated with high-dose co-trimoxazole intravenously and remained clinically well. He was also treated with anti-reflux

\begin{tabular}{lccc} 
TABLE 2 & Cell differential for bronchoalveolar lavage \\
& Cells counted $\mathbf{n}$ & $\%$ & Normal range \% \\
\hline Macrophages & 212 & 70.7 & $>80$ \\
Lymphocytes & 9 & 3.0 & $\leqslant 14$ \\
Neutrophils & 60 & 20.0 & $\leqslant 4$ \\
Eosinophils & 2 & 0.7 & $\leqslant 3$ \\
Mast cells & 0 & 0 & $\leqslant 0.5$ \\
\hline
\end{tabular}

medication. The patient was transferred to the care of a paediatric immunology team and genetic tests confirmed $\mathrm{X}$ linked SCID (T-B+NKC-). He underwent an extracorporeal gene therapy procedure and is currently doing well. There have been no further respiratory episodes that might have suggested aspiration in the 18 months of follow-up.

\section{DISCUSSION}

This patient was initially thought to have a lobar pneumonia, albeit with a rather prolonged course, and was nearly sent home because he had improved and been removed from treatment with oxygen. The case illustrates the following lessons: 1) PCP may mimic lobar pneumonia, at least on chest radiographs; 2) PCP may appear to improve even without specific treatment; 3) at presentation of SCID, there may be a normal lymphocyte count, and not lymphopenia, as described elsewhere [1].

PCP is an important cause of morbidity and mortality in immunocompromised patients, and is often the presenting feature in children with HIV infection [2]. In the UK, it remains the leading cause of admission to an intensive care unit in HIVpositive children [3]. PCP is also found in children with innate immunodeficiency, such as SCID, and due to iatrogenic immunosuppression following chemotherapy or transplantation [4].

SCID is a rare clinical syndrome characterised by recurrent and potentially life-threatening infections. A number of different molecular defects give rise to impaired or absent T-cells and associated direct or indirect B-cell dysfunction. A white cell differential will normally show a low lymphocyte count (typically $<2.8 \times 10^{9} \cdot \mathrm{L}^{-1}$ ) [1], although this was not a feature

\section{TABLE 3 Immunological investigations}

\begin{tabular}{|c|c|}
\hline $\lg G \mathbf{g} \cdot \mathrm{L}^{-1}$ & \\
\hline Total & $<2.0(3.1-13.8)$ \\
\hline $\lg _{1}$ & $<1.5(2.3-5.8)$ \\
\hline $\operatorname{lgG}_{2}$ & $<0.5(0.3-2.9)$ \\
\hline $\lg _{3}$ & $<0.1(0.1-0.8)$ \\
\hline $\operatorname{lgG}_{4}$ & $<0.1(0-0.5)$ \\
\hline $\lg A \mathrm{~g} \cdot \mathrm{L}^{-1}$ & $<0.1(0.3-1.2)$ \\
\hline $\lg M g \cdot L^{-1}$ & $0.3(0.5-2.2)$ \\
\hline $\operatorname{IgE} I U \cdot L^{-1}$ & $<0.5(0-29)$ \\
\hline Anti-tetanus IgG total IU $\cdot \mathrm{mL}^{-1}$ & 0.01 \\
\hline Diptheria toxoid $\mathrm{Ab} \mathrm{IU} \cdot \mathrm{mL}^{-1}$ & $<0.01$ \\
\hline WCC $10^{9} \cdot \mathrm{L}^{-1}$ & 7.82 \\
\hline Lymphocyte count $10^{9} \cdot \mathrm{L}^{-1}$ & 4.2 \\
\hline CD3 & $0.1 \%$ \\
\hline CD19 & $99.0 \%$ \\
\hline CD16+CD56+ & $0.2 \%$ \\
\hline CD3+CD56+ & $0.0 \%$ \\
\hline $\mathrm{CD} 3+\mathrm{CD} 4+$ & $0.0 \%$ \\
\hline CD3-CD4+ & $1.0 \%$ \\
\hline $\mathrm{CD} 3+\mathrm{CD} 8+$ & $0.0 \%$ \\
\hline CD3-CD8+ & $0.3 \%$ \\
\hline
\end{tabular}

Data ranges are given in parentheses. Ig: immunoglobulin; Ab: antibody; WCC white cell count. 
of the present case. Diagnosis is made by evaluating lymphocyte subsets and can be confirmed with specific genetic probes [5].

Children classically present with SCID aged 3-6 months, the majority of whom have respiratory infections. PCP has been reported as the presenting illness in $\sim 20 \%$ of children [6], although the true proportion is likely to be higher. In adults, induced sputum is a sensitive technique for diagnosing PCP [7], but this is clearly more difficult in children and nasopharyngeal specimens are not sensitive enough [8]. It may also coexist with respiratory viruses. BAL is the most reliable way of detecting PCP in infants, although an open lung biopsy may also be occasionally needed [9]. In the present case, the diagnosis was not made from repeated NPA nor oropharyngeal samples, including early morning gastric washouts.

Classically, PCP presents in young infants and toddlers with cough, fever, respiratory distress and hypoxaemia. The fever is often low grade, in contrast to the high fevers associated with bacterial infection, but the hypoxaemia and respiratory distress can be severe [10]. Focal signs are uncommon and are more frequently associated with bacterial infection [11]. Here, the patient had conflicting signs, with lobar changes but no fever, despite significant respiratory distress. Confusingly, he improved clinically without receiving specific treatment for PCP.

Chest radiographs will typically show diffuse reticular interstitial shadowing, especially prominent in the hilar regions. However, as with clinical signs, they may be normal in up to $30 \%$ of cases. HRCT scans are more sensitive and classically show a widespread ground-glass appearance in up to $90 \%$ of patients [12]. The changes may appear in mosaic fashion, with areas of unaffected lung adjoining affected areas. The pulmonary architecture is normally intact.

Unilateral changes, although uncommon, do not exclude a diagnosis of PCP. One review of 300 radiographs of patients with confirmed diagnosis of PCP found atypical presentations in $32 \%$, with unilateral signs in $11 \%$ [13], and a similar percentage of chest radiographs with focal abnormalities was found in a series of paediatric patients with HIV and PCP [14]. Cystic changes, cavitations, pleural effusions and pneumothoraces may also occur occasionally. Radiological improvement will often lag behind clinical improvement.

Treatment for children with PCP is high-dose i.v. co-trimoxazole $\left(60 \mathrm{mg} \cdot \mathrm{kg}^{-1}\right.$ b.i.d.) for 2-3 weeks, followed by oral prophylactic doses. Some centres advocate the use of steroids as adjunctive treatment [9]. SCID is treated in the short term with prophylactic antibiotics, vigorous treatment of infection and regular Ig therapy. Bone marrow transplant (ideally human leukocyte antigen (HLA)-matched) is the current definitive cure, ideally with a HLA-matched relative. Transplants are most successful when undertaken early in life [15]. However, clinical success and an improving safety profile in the use of gene therapy may dictate future treatment $[16,17]$.

Historically, PCP had a high mortality rate in paediatric patients, perhaps relating to the problems of diagnosis in children. In recent reviews, the outcome for children with SCID and coexistent PCP has dramatically improved [15].
However, respiratory infections at the time of presentation are still associated with a worse outcome [8]. In HIV, the finding of PCP at presentation also carries a poorer prognosis, especially in infants aged $<12$ months [18].

PCP is often thought of as a severe disease with classically diffuse chest signs and radiographical changes. Nonetheless, this case illustrates some important learning points. PCP may mimic (or coexist with) other respiratory infections, such as lobar pneumonia. Improvement can occur with conventional antibiotic treatment, although continuing signs, such as tachypnoea, suggest persisting disease. Normal initial investigations, such as white cell differential count, may suggest immunocompetence but cannot exclude abnormal immune function. Community-acquired pneumonia is a common presentation to acute paediatric units, but if it occurs in the presence of other abnormalities or concerns, such as diarrhoea and failure to thrive, underlying diseases, in particular immunodeficiency, should be considered. Unusual features, such as the presence of significant respiratory distress and radiographical changes with no fever or raised inflammatory markers, should also prompt further consideration of bronchoscopy and BAL.

Diagnosing Pneumocystis jiroveci pneumonia is a difficult challenge for respiratory paediatricians but a significant one to make, as underlying immunodeficiency must always be sought and actively treated.

\section{REFERENCES}

1 Hague RA, Rassam S, Morgan G, Cant AJ. Early diagnosis of severe combined immunodeficiency syndrome. Arch Dis Child 1994; 70: 260-263.

2 Shah I. Age related clinical manifestations of HIV in Indian children. J Trop Pediatr 2005; 51: 300-303.

3 Cooper S, Lyall H, Walters S, et al. Children with human immunodeficiency virus admitted to a paediatric intensive care unit in the United Kingdom over a 10-year period. Intensive Care Med 2004; 30: 113-118.

4 Gupta RK, Jain M, Garg R. Pneumocystis carinii pneumonia after renal transplantation. Indian J Pathol Microbiol 2004; 47: 474-476.

5 Buckley RH, Schiff RI, Schiff SE, et al. Human severe combined immunodeficiency: genetic, phenotypic, and functional diversity in one hundred eight infants. J Pediatr 1997; 130: 378-387.

6 Martinez Marin MI, de la Mota Ybancos JL, Romero Garcia J, et al. [Acute respiratory failure secondary to Pneumocystis carinii pneumonia in children.] An Esp Pediatr 1996; 45: 570-574.

7 Masur H, Lane HC, Kovacs JA, Allegra CJ, Edman JC. Pneumocystis pneumonia: from bench to clinic. Ann Intern Med 1989; 11: 813-826.

8 Berrington JE, Flood TJ, Abinun M, Galloway A, Cant AJ. Unsuspected Pneumocystis carinii pneumonia at presentation of severe primary immunodeficiency. Arch Dis Child 2000; 82: 144-147.

9 Gennery AR, Cant AJ. Diagnosis of severe combined immunodeficiency. J Clin Pathol 2001; 54: 191-195.

10 Selwyn PA, Pumerantz AS, Durante A, et al. Clinical predictors of Pneumocystis carinii pneumonia, bacterial 
pneumonia and tuberculosis in HIV-infected patients. AIDS 1998; 12: 885-893.

11 Graham SM, Mtitimila EI, Kamanga HS, Walsh AL, Hart CA, Molyneux ME. Clinical presentation and outcome of Pneumocystis carinii pneumonia in Malawian children. Lancet 2000; 355: 369-373.

12 Crans CA, Boiselle PM. Imaging features of Pneumocystis carinii pneumonia. Crit Rev Diagn Imaging 1999; 40: 251-284.

13 Cornalba GP, Mariani P, Colombo E, Bianco R, Vona S. Atypical radiological images in Pneumocystis carinii infection in HIV-positive patients. Radiol Med (Torino) 1994; 87: 763-767.

14 Sivit CJ, Miller CR, Rakusan TA, Ellaurie M, Kushner DC. Spectrum of chest radiographic abnormalities in children with AIDS and Pneumocystis carinii pneumonia. Pediatr Radiol 1995; 25: 389-392.

15 Fischer A, Landais P, Friedrich W, et al. European experience of bone marrow transplantation for severe combined immunodeficiency. Lancet 1990; 336: 850-854.

16 Ariga T. Gene therapy for primary immunodeficiency diseases: recent progress and misgivings. Curr Pharm Des 2006; 12: 549-556.

17 Fischer A, Hacein-Bey-Abina S, Lagresle C, Garrigue A, Cavazana-Calvo M. [Gene therapy of severe combined immunodeficiency disease: proof of principle of efficiency and safety issues.] Bull Acad Natl Med 2005; 189: 779-785.

18 Ikeogu MO, Wolf B, Mathe S. Pulmonary manifestations in HIV seropositivity and malnutrition in Zimbabwe. Arch Dis Child 1997; 76: 124-128. 